\title{
活性炭纤维的分形孔径分布及对低浓度 苯蒸气的吸附
}

黄正宏 康飞宇* 梁开明 杨骏兵

(清华大学材料科学与工程系, 北京 100084)

\begin{abstract}
摘要 在 Jaroniec 提出的 $J(x)$ 分形孔径分布函数基础上提出了 $K(x)$ 分形孔径分布函数, 并利用这 2 个函数对 3 种不同比表面积的活性炭纤维进行了表征; 通过非极性苯蒸气在 活性炭纤维上的等温线与分形孔径分布的比较研究, 揭示了活性炭纤维的分形孔径分 布与非极性苯在活性炭纤维上的等温线的关系.
\end{abstract}

\section{关键词活性炭纤维 分形 孔径分布 气相吸附}

活性炭纤维比表面积大, 微孔含量丰富, 孔径分布窄, 吸脱行程短, 具有优异的吸附性能 和吸附动力学行为, 非常适合于气相吸附, 特别适合于吸附质浓度较低时 ${ }^{[1 \sim 3]}$, 如室内空气净 化. 表征多孔炭材料的一个关键问题是对微孔隙的评价, 它影响和决定着吸附性质. 狭缝孔是 结构不规则性和能量不均匀性的主要来源, 可以用孔径分布进行定量表征, 但是对表面与结 构的不均匀性的描述还不够系统深人, Pfeifer 和 Avnir ${ }^{[4]}$ 把分形的概念引人到多孔材料中, 从而 可以定量描述多孔固体表面的结构不规则性和能量不均匀性. 几乎所有的高比表面积的固体 都具有 $2 \sim 3$ 的分形维数 (通常用 $D$ 表示), $D$ 值越接近于 2 , 则表面越光滑; 而 $D$ 值越接近于 3 , 则 表面越粗粘. 本文利用氮气吸附研究了黏胶基活性炭纤维的表面分形 ${ }^{[5,6]}$, 并用重量法测得大 气压、室温条件下低浓度苯蒸气的吸附等温线, 探讨了分形特征和苯蒸气吸附之间的关系.

\section{1 理论基础}

Jaroniec 等人 ${ }^{[7]}$ 提出了分形维数为 $D$ 的多孔固体的孔径分布函数如下

$$
J(x)=\frac{3-D}{x_{\max }^{3-D}-x_{\min }^{3-D}} x^{2-D},
$$

其中 $x$ 为狭缝孔半宽. 推导如下: 根据微孔吸附理论, 吸附等温线可表示为

$$
\Theta=\int \theta J(x) \mathrm{d} x,
$$

$\Theta$ 为总吸附等温线, $\theta$ 为局部吸附等温线, $J(x)$ 为 $\left(x_{\min }, x_{\max }\right.$ 范围的微孔孔径分布. 对具有分形 表面的微孔固体, 孔径分布函数 $J(x)$ 满足如下比例关系

$$
J(x)=\rho x^{2-D},
$$

$\rho$ 为比例常数. 将微孔孔径分布归一化 


$$
\int_{x_{\min }}^{x_{\max }} J(x) \mathrm{d} x=1,
$$

则得到分形微孔固体的分形孔径分布, 如(1)式所示.

另外, 可以得到与 $J(x)$ 有关的平均孔径如下 ${ }^{[8]}$

$$
\bar{x}=x_{\min }\left(\frac{3-D}{4-D}\right)\left(\frac{r^{4-D}-1}{r^{3-D}-1}\right),
$$

其中 $r=x_{\max } / x_{\min }$.

如果将 $\Theta=V / V_{0}$ 带人(2)式, 则

$$
V=\int_{x_{\min }}^{x_{\max }} V_{0} \vartheta J(x) \mathrm{d} x,
$$

$V_{0}$ 为吸附剂微孔容积, $V$ 为吸附质占据的微孔容积. 令 $K(x)=V_{0} J(x)$, 则

$$
\int_{x_{\min }}^{x_{\max }} K(x) \mathrm{d} x=V_{0},
$$

可以得到分形微孔固体改进后的分形孔径分布

$$
K(x)=V_{0} \frac{3-D}{x_{\max }^{3-D}-x_{\min }^{3-D}} x^{2-D} .
$$

从得到的结果看, (8)和(1)式虽然没有实质性的差别, 但是(1)式反映的是分形微孔固体的 不同尺度孔的比例，而改进后(8)式反映了不同尺度孔的相对孔容值. 下面用改进前后的分形 孔径分布函数对吸附实验进行讨论.

\section{2 实验}

3 种表面积不同的黏胶基活性炭纤维(由鞍山市活性炭纤维厂提供), 在 $360 \mathrm{CX}$ 氮气吸附仪 (美国库尔特公司)上 $77 \mathrm{~K}$ 下进行等温吸附. 样品在 $250^{\circ} \mathrm{C}, 6 \mathrm{e}-5 \times 13.332 \mathrm{~Pa}$ 条件下脱气 $12 \mathrm{~h}$. 样 品按 BET 比表面积依次标记为 ACF6, ACF14 和 ACF16.

将 10 20 mg 活性炭纤维样品放人热重分析仪(美国 TA 公司)托盘上, 在 $60 \mathrm{~cm}^{3} / \mathrm{min}$ 高纯 氮气吹扫下升温至 $120^{\circ} \mathrm{C}$, 保温 $1 \mathrm{~h}$ 以去除水分及其他物理吸附的物质, 然后降温至 $25^{\circ} \mathrm{C}$, 保 持一段时间后切换为低浓度的苯蒸气. 苯蒸气采用标准气体(北京氦普北分气体公司), 用高纯 氮气稀释到低浓度. 整个气路的流速用质量流量控制器(北京建中机器厂)进行控制.

\section{3 结果与讨论}

\section{1 活性炭纤维的孔隙结构和分形特征}

$\mathrm{N}_{2}$ 吸附等温线结果表明 ${ }^{[6]}: 3$ 种样品为微孔吸附剂. 从得到的孔结构参数看(见表 1), 随着 比表面积的增加, 其微孔容积也增加. 用 H-K 法对样品的微孔分布进行分析 ${ }^{[6]}$ : ACF6 的表面 积最小, 但有最为丰富的极微孔, ACF16 与 ACF14 相比其极微孔略显丰富一些. 从 H-K 平均 孔径大小看, ACF6 最小, ACF14 略大于 ACF16. 确定 $D$ 值的方法有吸附法、X 射线小角散射 法、电子显微镜分析的方法等等 ${ }^{[5]}$, 本文用氮气吸附得到样品的分形维数 (见表 1). ACF6 的比 表面积最小、微孔容积最低, 但是其分形维数却最高, 表明其孔表面最为粗粘, 即极微孔的含 量最高, 从表 I 可见其 H-K 平均孔径最小. ACF16 比表面积最大、微孔容积最高, 但其分形维 数并不是最低, 从 $\mathrm{H}-\mathrm{K}$ 孔径分布曲线看, 其峰略高于 $\mathrm{ACF} 14^{[6]}$. 另外, 从 $J(x)$ 得到的平均狭缝 
半宽与 $\mathrm{H}-\mathrm{K}$ 平均孔径结果较为一致.

表 1 活性炭纤维的孔隙与分形性质

\begin{tabular}{cccccc}
\hline 样品 & 比表面积 $/ \mathrm{m}^{2} \cdot \mathrm{g}^{-1}$ & 微孔容积 $/ \mathrm{cm}^{3} \cdot \mathrm{g}^{-1}$ & 分形维数 & $\mathrm{H}-\mathrm{K}$ 平均孔径 $/ \mathrm{nm}$ & $J(x)$ 平均狭缝半宽 $/ \mathrm{nm}$ \\
\hline ACF6 & 640 & 0.271 & 2.84 & 0.70 & 0.42 \\
ACF14 & 1460 & 0.451 & 2.26 & 0.96 & 0.51 \\
ACF16 & 1680 & 0.546 & 2.56 & 0.95 & 0.46 \\
\hline
\end{tabular}

按照文献[8], 假设 $x_{\text {max }}=1.0 \mathrm{~nm}, x_{\text {min }}=0.1 \mathrm{~nm}$, 将表 1 中 $D$ 值分别带人方程(1)和方程(8), 可以得到 $J(x)$ 和 $K(x)$ 分形孔径分布曲线(如图 1, 2 所示). 可见, $J(x)$ 和 $K(x)$ 都是单调递减函数. $J(x)$ 直观地反映了一般分形多孔固体中极微孔和超微孔的比例分配(按照国际与应用化学会 (IUPAC)的分类 ${ }^{[9]}$, 孔宽度小于 $2.0 \mathrm{~nm}$ 为微孔, 进一步可以分为, 孔宽度小于 $0.7 \mathrm{~nm}$ 为极微孔, 介于 $0.7 \sim 2 \mathrm{~nm}$ 之间的为超微孔 ${ }^{[10]}$ ), $D$ 值越大、较小微孔(极微孔)部分分布曲线越高, 即极微孔 所占比例越高, 则表面越粗䊁. $D$ 值越小, $J(x)$ 曲线越平坦, 则多孔固体表面的结构规则性和能 量均匀性越好. 如果将分形固体的微孔容积引人, 则分布曲线发生了移动, 得到分形多孔固体的 $K(x)$ 孔径分布, 曲线的陡峭程度反映了分形维数的大小, 而曲线的位置反映了微孔孔容的大小.

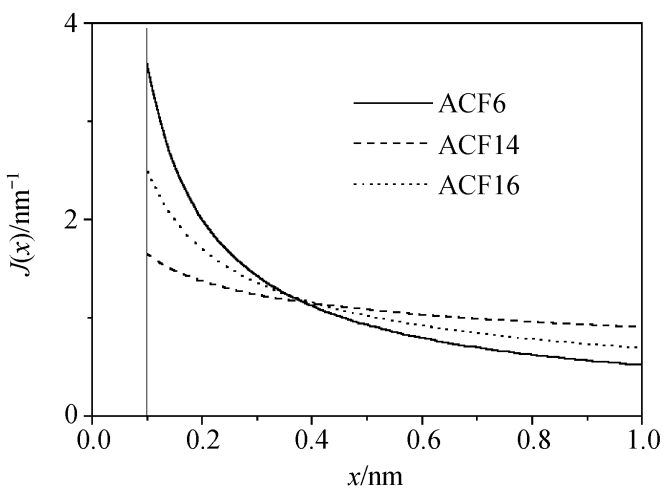

图 1 活性炭纤维的 $J(x)$ 分形孔径分布

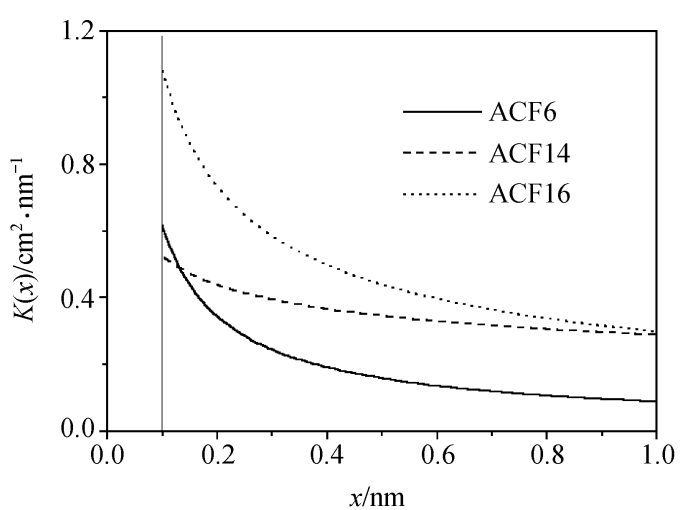

图 2 活性炭纤维的 $K(x)$ 分形孔径分布

\section{2 活性炭纤维对低浓度苯蒸气的吸附}

活性炭纤维由于其优异的吸附性能, 在室内空气中挥发性有机化合物(VOC)的控制方面 发挥着越来越重要的作用. 因为苯为非极性物质, 可以避免活性炭纤维表面官能团对吸附的

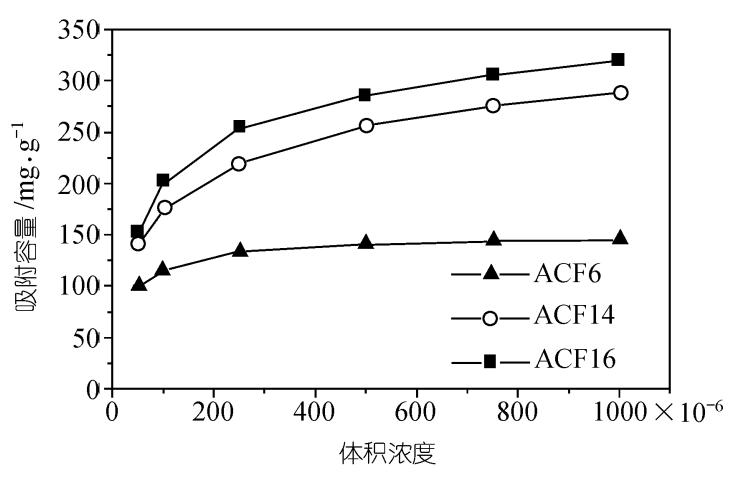

图 3 苯蒸气在活性炭纤维上的等温线 影响, 本文研究了活性炭纤维对 VOC 中较有 代表性的物质苯在室温 $25^{\circ} \mathrm{C}$ 和大气压条件下 的吸附, 并探讨了和活性炭纤维分形孔径分 布之间的关系.

由于低浓度的苯蒸气达到吸附平衡很慢, 例如在约 $50 \times 10^{-6}$ 浓度下需要 20 30 h, 因而 本文只测定了 50 1000 × 10 $0^{-6}$ 浓度范围的吸附 等温线(见图 3). 从图中可见, 在所测浓度范 围内, 比表面积越大, 吸附容量越高, 吸附等 温线位置越高. ACF14 与 ACF16 的比表面积、 
微孔容积比较接近, 因而两条等温线靠的很近; 而 ACF6 由于微孔容积最小, 吸附容量最低, 等温线较为平坦, 在较低的浓度下就能达到饱和. 我们将图 3 与 $K(x)$ 分形孔径分布曲线(超微 孔部分)相比较则可以看出, $K(x)$ 分形孔径分布曲线较高的样品, 其等温线的位置也较高. 由此 可见，在较高浓度下吸附时起作用的是微孔孔容.

Dubinin-Radushkevich 方程 ${ }^{[11]}$ 用来描述非 极性和弱极性物质在微孔吸附剂上的吸附, 和 实验结果吻合得很好. 我们用 D-R 方程对图 3 进行拟合，并将浓度范围扩大到 $0.1 \sim 10000 \times$ $10^{-6}$, 结果见图 4. 由图可见, 当苯蒸气在极低 浓度时, ACF6 的吸附容量最高. 这是因为微孔 临近孔壁的势场互相叠加, 增加了固体与气体 分子的相互作用能, 在低一些的压力下发生小 一些的微孔容积充填, 且孔径越小, 这种增强

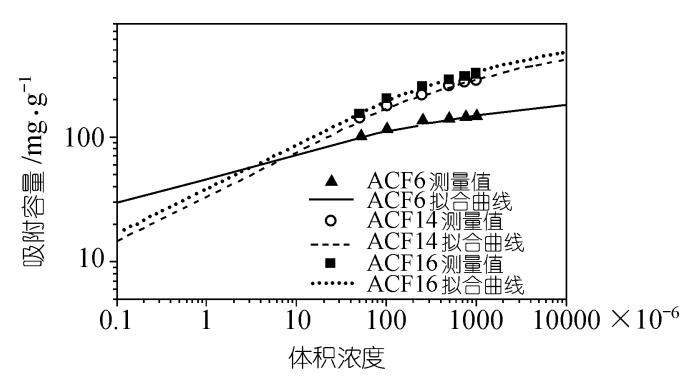

图 4 D-R 方程对苯蒸气在活性炭纤维上的吸附 等温线的拟合

作用越强 ${ }^{[9]}$. ACF6 的微孔孔径分布最窄、平均孔径最小, 因而 ACF6 对极低浓度的苯蒸气的吸 附容量最高. 我们将拟合曲线与 $J(x)$ 分形孔径分布曲线(极微孔部分)相比较则可以看出, $J(x)$ 分 形孔径分布曲线较高的样品, 其等温线在极低浓度部分的位置也较高. 可以这样说, 分形维数 越高的微孔吸附剂，对极低浓度的非极性物质的吸附容量越高.

\section{4 结论}

$J(x)$ 函数表征了分形多孔固体的不同尺度孔径的比例, 而改进后的 $K(x)$ 函数表征了分形 多孔固体不同尺度孔的相对孔容. 通过 2 个函数对非极性苯蒸气在活性炭纤维上的吸附等温 线的分析表明，不同浓度下吸附质的决定因素不同，较高浓度下吸附时起作用的是微孔孔容， 而较低浓度下起作用的是极微孔的比例. $J(x)$ 和 $K(x)$ 分别反映了活性炭纤维在极低吸附质浓度 和较高吸附质浓度下的吸附特性.

\section{参考文献}

1 Foster K L, Fuerman R G, Economy J, et al. Adsorption characteristics of trace volatile organic compounds in gas streams onto activated carbon fibers. Chemistry of Materials, 1992, 4(5): 1068 1073

2 Huang Z H, Kang F Y, Liang K M. Adsorpton of VOCs on viscose rayon based activated carbon fiber. In: Scharff P, ed. The Proceedings of $1^{\text {st }}$ World Conference on Carbon, 2000. Berlin: German Carbon Group, 2000. 143 144

3 Suzuki M. Activated carbon fiber: fundamentals and applications. Carbon, 1994, 32(4): 577 586

4 Pfeifer P, Avnir D. Chemistry in noninteger dimensions between two and three. I. Fractal theory of heterogeneous surfaces. J Chem Phys, 1983, 79(7): 3558 3565

5 Ismail I M K, Pfeifer P. Fractal analysis and surface roughness of nonporous carbon fibers and carbon blacks. Langmuir, 1994, 10: 1532 1538

6 黄正宏, 康飞宇, 梁开明, 等. 用氮气吸附研究活性炭纤维的分维. 炭素技术, 2000, (4): 40 42

7 Jaroniec M, Lu X, Madey R, et al. Thermodynamics of gas adsorption on fractal surfaces of heterogeneous microporous solids. J Chem Phys, 1990, 92(12): 7589 7595

8 Jaroniec M, Gilpin R K. Correlation between microporosity and fractal dimension of active carbons. Carbon, 1993, 31(2): $325 \sim 331$

9 Gregg S J, Sing K S W. Adsorption, Surface Area and Porosity. London: Academic Press, 1982. 218 257

10 Kaneko K, Setoyama, Suzuki T. Ultramicropore charaterization by He adsorption. Stud Surf Sci Catal, 1994, 87: 593 602

11 Dubinin M M. Fundamentals of the theory of adsorption in micropores of carbon adsorbents: Characteristics of their adsorption properties and microporous structures. Carbon, 1989, 27: 457 467 